\title{
Ultrafast Extreme Ultraviolet Induced Isomerization of Acetylene Cations
}

\author{
Y. H. Jiang, ${ }^{1}$ A. Rudenko, ${ }^{2}$ O. Herrwerth, ${ }^{3}$ L. Foucar, ${ }^{2}$ M. Kurka, ${ }^{1}$ K. U. Kühnel, ${ }^{1}$ M. Lezius, ${ }^{3}$ M. F. Kling, ${ }^{3}$ J. van Tilborg, ${ }^{4}$ \\ A. Belkacem, ${ }^{4}$ K. Ueda, ${ }^{5}$ S. Düsterer ${ }^{6}$ R. Treusch, ${ }^{6}$ C. D. Schröter, ${ }^{1}$ R. Moshammer, ${ }^{1}$ and J. Ullrich ${ }^{1,2}$ \\ ${ }^{1}$ Max-Planck-Institut für Kernphysik, 69117 Heidelberg, Germany \\ ${ }^{2}$ Max-Planck Advanced Study Group at CFEL, 22607 Hamburg, Germany \\ ${ }^{3}$ Max-Planck-Institut für Quantenoptik, 85748 Garching, Germany \\ ${ }^{4}$ Lawrence Berkeley National Laboratory, Berkeley, California 94720, USA \\ ${ }^{5}$ Institute of Multidisciplinary Research for Advanced Materials, Tohoku University, 980-8577 Sendai, Japan \\ ${ }^{6}$ DESY, 22607 Hamburg, Germany
}

(Received 11 August 2010; published 22 December 2010)

\begin{abstract}
Ultrafast isomerization of acetylene cations $\left([\mathrm{HC}=\mathrm{CH}]^{+}\right)$in the low-lying excited $A^{2} \Sigma_{g}{ }^{+}$state, populated by the absorption of extreme ultraviolet (XUV) photons $(38 \mathrm{eV})$, has been observed at the Free Electron Laser in Hamburg, (FLASH). Recording coincident fragments $\mathrm{C}^{+}+\mathrm{CH}_{2}{ }^{+}$as a function of time between XUV-pump and -probe pulses, generated by a split-mirror device, we find an isomerization time of $52 \pm 15$ fs in a kinetic energy release (KER) window of $5.8<\mathrm{KER}<8 \mathrm{eV}$, providing clear evidence for the existence of a fast, nonradiative decay channel.
\end{abstract}

PACS numbers: $31.70 . \mathrm{Hq}, 33.80 . \mathrm{Gj}, 41.60 . \mathrm{Cr}, 82.30 . \mathrm{Qt}$

Isomerization is an elementary chemical reaction occurring in molecules as well as in biological functional complexes, where it often triggers important biochemical reactions, e.g., in photosynthesis or eye vision [1]. As in other reactions, a whole sequence of transient species, the transition states, with intermediate geometries might be involved [2]. In general, it has become a dream of chemical physicists to "watch" geometrical rearrangements during chemical reactions and to observe transition states in real time. Recently, the development of near infrared (IR) pump-extreme ultraviolet (XUV) probe (or vice versa) techniques holding the promise of achieving attosecond time resolution [3] has started providing detailed insight into the time evolution of molecular reactions [4]. Moreover, ultrafast electron diffraction, now touching the femtosecond (fs) regime, or intense fs pulses from x-ray free electron lasers (FEL), just coming into operation, nurture the vision to visualize structural changes during molecular reactions as a function of time, i.e., to "make the molecular movie."

Acetylene $\left(\mathrm{C}_{2} \mathrm{H}_{2}\right)$ has been serving as a prototype for studying isomerization [5-15]. Upon absorption of a XUV photon, the $\mathrm{H}$ atom in $[\mathrm{HC}=\mathrm{CH}]^{+}$might migrate from one $\mathrm{C}$ atom to the other via trans-bent modes within a certain time and, consequently, the linear $\left(\mathrm{D}_{\infty h}\right)$ acetylene cation $[\mathrm{HC}=\mathrm{CH}]^{+}$rearranges into the nonlinear $\left(\mathrm{C}_{2 v}\right)$ vinylidene $\left[\mathrm{H}_{2} \mathrm{CC}\right]^{+}$structure. The $\mathrm{CH}_{2}{ }^{+}$fragment resulting from isomerization has been detected previously in dissociative photoionization [8] or electron-impact ionization [9] below the double ionization threshold, but, up to now, studies devoted to explore the time evolution of isomerization in $[\mathrm{HC}=\mathrm{CH}]^{+}$have not been reported. Particularly attractive is the decay of the acetylene cation in the excited ionic state $\left(A^{2} \Sigma_{g}{ }^{+}\right)$. Here, neither the absorption nor emission bands of the dipole-allowed $A^{2} \Sigma_{g}{ }^{+} \rightarrow X^{2} \Pi_{u}$ transition to the ground state have been detected $[5,6,16]$, and, due to the existence of high potential barriers, the excited level cannot dissociate directly into the $\mathrm{H}^{+}+\mathrm{C}_{2} \mathrm{H}$ or $\mathrm{H}+\mathrm{C}_{2} \mathrm{H}^{+}$channels. However, the autocorrelation function derived from photoelectron spectra depicted an ultrafast relaxation of the $A^{2} \Sigma_{g}{ }^{+}$state of 51 fs [7]. Theoretical studies suggested an efficient coupling mechanism allowing for an ultrafast redistribution of energy via isomerization $[5,6,11]$ or vibronic coupling [12]. Because of the absence of time-resolved benchmark experiments, the question on the dynamics responsible for the ultrafast, nonradiative decay of $A^{2} \Sigma_{g}{ }^{+}$has remained open and debated for more than three decades.

In this Letter we report on tracing ultrafast isomerization of $[\mathrm{HC}=\mathrm{CH}]^{+}$in the first excited $A^{2} \Sigma_{g}{ }^{+}$state in real time by combining three forefront technologies: (i) the Free Electron Laser in Hamburg (FLASH) [17], delivering XUV photons with few-femtosecond time resolution and unprecedented intensities, (ii) multihit coincident fragment imaging methods along with (iii) a newly developed splitmirror pump-probe setup. The $A^{2} \Sigma_{g}{ }^{+}$state can be effectively populated by ionization from the neutral acetylene molecule by absorption of a $38 \mathrm{eV}$ photon in the pump pulse [18]. Analyzing the dissociation channel $\left[\mathrm{CCH}_{2}\right]^{2+} \rightarrow \mathrm{C}^{+}+\mathrm{CH}_{2}{ }^{+}$induced by the probe pulse as function of the pump-probe delay time we are able to record the isomerization time, where the $\mathrm{CH}_{2}{ }^{+}$fragment serves as an indicator for the migration of the $\mathrm{H}$ atom.

Our experiment, a reaction microscope (REMI) [19] equipped with an on-axis backreflection split-mirror arrangement for focusing and pulse-pair creation, was installed at beam line BL 3 of FLASH. Our device consists of a spherical multilayer mirror $(1 \mathrm{in}$. Mo/Si mirror, $50 \mathrm{~cm}$ 
focal length) that is cut into two identical "half-mirrors" (so called "half-moon" geometry). It has a reflectivity of $40 \%$, sharply peaked around $38 \mathrm{eV}$ such that higher order harmonic radiation from the FEL is efficiently suppressed by 3- 4 orders of magnitude to a negligibly low level. While one half-mirror is mounted at a fixed position, the other one is movable along the FEL beam axis by means of a high-precision piezostage. In this way the time delay between both reflected light pulses is adjustable within a range of $\pm 2500 \mathrm{fs}$ at a resolution of better than $1 \mathrm{fs}$. The time overlap of both pulses was determined by detecting the delay dependence of coincident fragments $\mathrm{C}^{2+}+\mathrm{C}^{2+}$, where maximum in the yield at zero delay time was observed [Fig. 3(c)]. It provides us with the time-zero information and indicates that structures below 20 fs can be resolved. In a separate measurement, just done before under the same experimental conditions, time-dependent features of less than $10 \mathrm{fs}$ [20] were observed when mapping the ultrafast oscillatory motion $(\sim 22 \mathrm{fs})$ of the nuclear wave packet in $\mathrm{D}_{2}^{+}\left(1 s \sigma_{g}\right)$. For a more detailed discussion on the FLASH time structure and time resolutions achievable, see [21,22].

During the experiment, the intensity of the incoming FEL beam $(10 \mathrm{~mm}$ diameter at the position of the backreflecting focusing mirror) was equally distributed over both half-mirrors and the foci were merged inside a dilute and well-localized beam (less than $1 \mathrm{~mm}$ diameter) of cold $\mathrm{C}_{2} \mathrm{H}_{2}$ molecules in the center of REMI. With a focus diameter of $\sim 10 \mu \mathrm{m}$ and pulse energies of a few $\mu \mathrm{J}$ at an estimated average pulse duration of $\sim 30 \mathrm{fs}$ (see also $[21,22])$, we reached peak intensities of $I \cong$ $10^{13}-10^{14} \mathrm{~W} / \mathrm{cm}^{2}$ at $38 \pm 0.5 \mathrm{eV}$. Ionic fragments were projected by means of an electric field $(40 \mathrm{~V} / \mathrm{cm})$ onto a time- and position-sensitive microchannel plate detector (diameter $120 \mathrm{~mm}$, position resolution $0.1 \mathrm{~mm}$, multihit delay-line readout) and recorded as a function of the pumpprobe time delay. From the measured time of flight (TOF) and position of each individual fragment, the initial threedimensional momentum vectors were reconstructed. The energy resolution in the KER spectra is better than $50 \mathrm{meV}$ for all fragment energies detected.

In Fig. 1 the transition pathways for isomerization from the acetylene to the vinylidene cation are illustrated, which can be expressed by the following reaction equations:

$$
\begin{aligned}
& \operatorname{HCCH}\left(X^{1} \Sigma_{g}^{+}\right)+h v(\text { pump }) \\
& \quad \rightarrow[\mathrm{HCCH}]^{+}\left(X^{2} \Pi_{u}, A^{2} \Sigma_{g}\right)+e_{1} \\
& \quad \stackrel{\text { migra }}{\longrightarrow}\left[\mathrm{H}_{2} \mathrm{CC}\right]^{+}\left(\tilde{X}^{2} B_{1}, \tilde{A}^{2} B_{2}\right)+e_{1},
\end{aligned}
$$

and

$$
\begin{aligned}
{\left[\mathrm{H}_{2} \mathrm{CC}\right]^{+}\left(\tilde{X}^{2} B_{1}, \tilde{A}^{2} B_{2}\right)+h v(\text { probe }) } & \rightarrow\left[\mathrm{H}_{2} \mathrm{CC}\right]^{2+}+e_{2} \\
& \stackrel{\text { diss }}{\longrightarrow} \mathrm{CH}_{2}^{+}+\mathrm{C}^{+}+e_{2},
\end{aligned}
$$

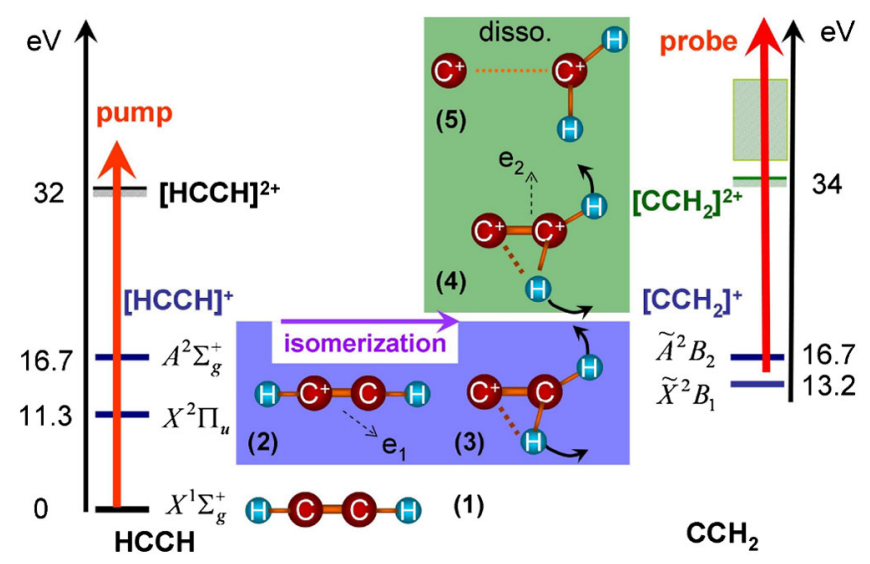

FIG. 1 (color online). Energy level diagrams for the acetylene (left) and vinylidene (right) cations as well as sketches of the expected isomerization-dissociation processes (middle). Numbers indicate possible time-sequenced stages of neutral acetylene (1), acetylene cation (2), transient states to vinylidene (3), ionization of the vinylidene cation (4), and Coulomb explosion of the vinylidene dication (5), respectively. Vertical arrows indicate photon absorptions. Energy levels were taken from Refs. [13,18,23].

with $e_{1}$ and $e_{2}$ being the first and second emitted electron, respectively. In expression (1) the absorption of one photon in the pump pulse leads to the formation of the acetylene cation $[\mathrm{HCCH}]^{+}$in the ground $X^{2} \Pi_{u}$ and the low-lying excited $A^{2} \Sigma_{g}$ state. The probability for populating the $X^{2} \Pi_{u}$ ground and the $A^{2} \Sigma_{g}{ }^{+}$dominate other channels by a factor of more than 3 for ionization at $38 \mathrm{eV}$ [18]. Here, on the $A^{2} \Sigma_{g}$ potential curve, $[\mathrm{HCCH}]^{+}$is unstable because of almost no potential barriers for isomerization to $\left[\mathrm{H}_{2} \mathrm{CC}\right]^{+}$in the $\tilde{X}^{2} B_{1}$ and $\tilde{A}^{2} B_{2}$ states as illustrated in Fig. 1. The $[\mathrm{HCCH}]^{+} \rightarrow\left[\mathrm{H}_{2} \mathrm{CC}\right]^{+}$isomerization is more closely related to the trans-bent and $\mathrm{CC}$ stretch motions than to the $\mathrm{CH}$ stretch motions, since during the course of the isomerization one hydrogen must move from one end (breaking of $\mathrm{CH}$ bond) to the other end of the $\mathrm{CC}$ core (making of $\mathrm{CH}$ bonds) and the $\mathrm{CC}$ bond might be varied considerably. This reaction leading to molecular rearrangement is now mapped in real time by measuring coincident fragments $\mathrm{CH}_{2}{ }^{+}+\mathrm{C}^{+} \leftarrow\left[\mathrm{H}_{2} \mathrm{CC}\right]^{2+}$ as a function of the delay time, where $\left[\mathrm{H}_{2} \mathrm{CC}\right]^{2+}$ is created by the probe pulse as described in expression (2) with the $\mathrm{CH}_{2}{ }^{+}$ isomer fragment being a unique signature for $\mathrm{H}$-atom transfer.

In Fig. 2 the TOF of the first fragment is plotted as a function of the TOF of the second one. Two diagonal lines are clearly discernible indicating two coincident fragmentation channels, namely, $\mathrm{CH}^{+}+\mathrm{CH}^{+}$and $\mathrm{CH}_{2}{ }^{+}+\mathrm{C}^{+}$ providing experimental evidence for the existence of isomerization of acetylene cations, different from previous Auger spectroscopy measurements [14], where both channels could not be distinguished. 


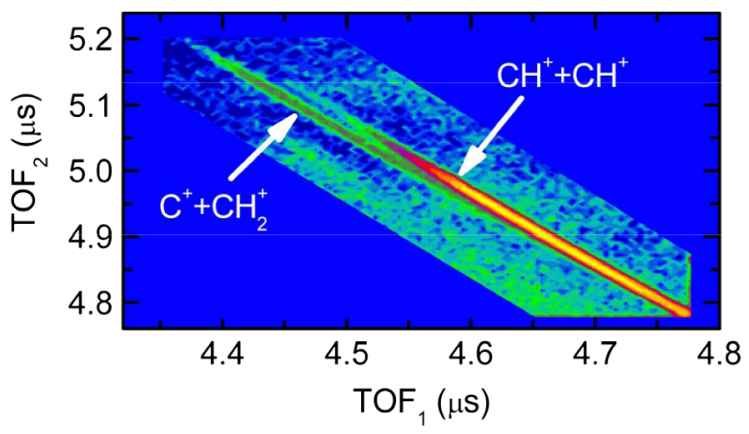

FIG. 2 (color online). Time of flight (TOF) of the first fragment versus TOF of second one. The data are integrated over all time delays $|\Delta t|<360$ fs.

The appearance thresholds for the production of $\mathrm{CH}^{+}+$ $\mathrm{CH}^{+}$(dissociation), $\mathrm{CH}_{2}^{+}+\mathrm{C}^{+}$(isomerizationdissociation), and $\mathrm{C}_{2} \mathrm{H}^{+}+\mathrm{H}^{+} \quad$ (deprotonationdissociation) are around $32-34 \mathrm{eV}$, which means that the isomerization-dissociation channel can be launched by the absorption of either one or two photons in a sequential way, leading to excess energies of about 4 and $22-27 \mathrm{eV}$, respectively (only considering the first two lowest states of the acetylene cation). Here, the sequential pathway is approximated as a "stepwise" absorption through the intermediate ionic states of $[\mathrm{HCCH}]^{+}$. The number of photons triggering the reaction in the actual experiment can be identified by inspecting the branching ratios among the three dissociation channels, which are very sensitive to the excess energy of the photoelectrons [23,24]. The here-observed ratios of $71 \%$ for $\mathrm{C}_{2} \mathrm{H}^{+}+\mathrm{H}^{+}, 25 \%$ for $\mathrm{CH}^{+}+\mathrm{CH}^{+}$, and $4 \%$ for $\mathrm{CH}_{2}^{+}+\mathrm{C}^{+}$(integrated over all delays) are in good quantitative agreement with theoretical predictions [23] for one-photon double ionization of acetylene at excess energies of about $20 \mathrm{eV}$. Here one can assume that one-photon double ionization should lead to similar fragmentation ratios as two-photon ionization at the same excess energy of $20 \mathrm{eV}$. This is very different from previous measurements with $38 \mathrm{eV}$ photons leading to small excess energies $(\sim 4 \mathrm{eV})$, where almost comparable probabilities were found for the production of $\mathrm{CH}_{2}{ }^{+}+\mathrm{C}^{+}$ and $\mathrm{CH}^{+}+\mathrm{CH}^{+}$. Therefore, the observed branching ratios are considered as direct evidence for the dominance of two-photon transitions to produce the doubly charged cation under present experimental conditions although onephoton double ionization is allowed energetically. A similar effect, a two-photon transition to a doubly charged molecular ion being more effective than the energetically possible one-photon absorption, was observed before in multiple ionization of $\mathrm{N}_{2}$ [25].

Applying strict momentum conservation conditions one can fully separate the $\mathrm{CH}_{2}{ }^{+}+\mathrm{C}^{+}$isomerization channel from the charge-symmetric $\mathrm{CH}^{+}+\mathrm{CH}^{+}$dissociation reaction, except for the events with $P_{\perp} \rightarrow 0$ a.u.. The present KER spectrum for coincident fragments $\mathrm{CH}_{2}{ }^{+}+\mathrm{C}^{+}$together with results from previous measurements in strong

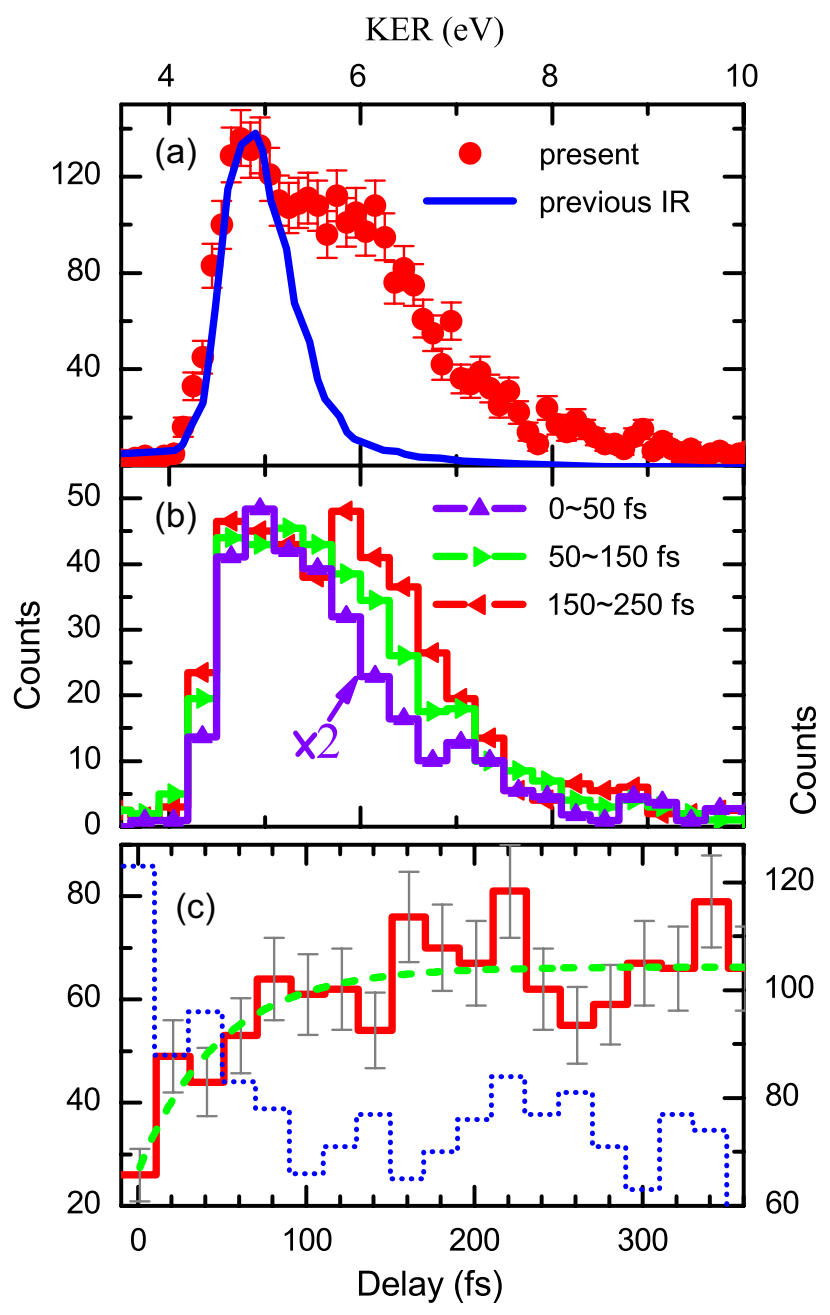

FIG. 3 (color online). Kinetic energy release (KER) and timedelay spectra for coincident $\mathrm{CH}_{2}{ }^{+}+\mathrm{C}^{+}$fragments. (a) KER distribution integrated at all delay times. Full circles, present data; solid line, strong IR laser pulses [26]. (b) KER spectra at different delay times integrated over $\pm 50 \mathrm{fs}$. (c) Yield of $\mathrm{CH}_{2}{ }^{+}+\mathrm{C}^{+}$coincident fragments (solid red line) for $5.8<$ $E_{\mathrm{KER}}<8 \mathrm{eV}$ in (a). Dashed green line, fit of exponential function; dotted blue line, autocorrelation trace for coincident $\mathrm{C}^{2+}+$ $\mathrm{C}^{2+}$ fragments (right $y$ axis).

IR field [26] are presented in Fig. 3(a). Two contributions peaked at $E_{\mathrm{KER}} \cong 4.8$ and $5.8 \mathrm{eV}$, respectively, can be identified. The isomerization leading to lower KERs is found to be time independent, whereas the high energy tail, not observed in previous measurements, shows a clear evolution as a function of time as shown in Fig. 3(b) where the KER spectra at three delay times around 0, 100, and $200 \mathrm{fs}$ are presented (a \pm 50 fs wide integration range was chosen due to limited statistics at a FLASH pulse repetition rate of $150 \mathrm{~Hz}$ leading to $0.1 \mathrm{~Hz}$ coincident events for the relevant channel). The time-independent low-KER part peaked at $4.8 \mathrm{eV}$ is due to sequential two-photon absorption through intermediate states of the acetylene cation, populating the dication within a single pulse, either the pump or the probe, and subsequent isomerization yielding $\mathrm{CH}_{2}{ }^{+}+\mathrm{C}^{+}$ 
fragments. The present KER peak position is consistent with the one observed for acetylene dication isomerization in a strong IR field [26] [full line in Fig. 3(a)]. The $\mathrm{C}_{2} \mathrm{H}_{2}{ }^{2+}$ configuration in the first triplet electronic state of $1^{3} \Pi$, $5.3 \mathrm{eV}$ above the ground state of $\mathrm{C}_{2} \mathrm{H}_{2}{ }^{2+}$, is assumed to be unstable and has been speculated to be responsible for acetylene-vinylidene isomerization of the dication $[15,23]$.

Remarkably, the peak at high KERs $(\cong 5.8 \mathrm{eV})$ in Fig. 3(a) was absent in previous studies of dication isomerization in IR fields [26] and Auger spectroscopy [14] studies and, thus, represents a novel rearrangement pathway. Interestingly, it displays a clear time dependence shown in Figs. 3(b) and 3(c), indicating the migration of the $\mathrm{H}$ atom in the acetylene cation. As described in expressions (1) and (2), the $\mathrm{H}$ atom starts to proceed a trans-bent motion in the unstable ionic $A^{2} \Sigma_{g}$ states of $[\mathrm{HCCH}]^{+}$created by the pump pulse and mapped by the probe pulse via coincident $\mathrm{CH}_{2}{ }^{+}+\mathrm{C}^{+}$fragment detection as a function of the delay time [Fig. 3(c)]. We find an effective isomerization time of $52 \pm 15 \mathrm{fs}$ by fitting an exponential function to the data for all KERs between 5.8 and $8 \mathrm{eV}$. This value is in very good agreement with the nonradiative lifetime (51 fs) of the $A^{2} \Sigma_{g}{ }^{+}$state derived in time-independent experiments from the correlation function [7]. Therefore, our study presents experimental evidences that the rapid decay mechanism of $A^{2} \Sigma_{g}{ }^{+}$state observed before and discussed in the literature is attributed to isomerization $[5,6,11]$, and not to a conical intersection with the $\tilde{X}^{2} \Pi_{u}$ state [12].

Conclusive evidence that the $A^{2} \Sigma_{g}{ }^{+}$state is populated and studied is given by the facts that (i) the probability for populating the $X^{2} \Pi_{u}$ ground and the $A^{2} \Sigma_{g}{ }^{+}$excited states of the acetylene cation dominate other channels by a factor of more than 3 as mentioned before. Moreover, there is a significant potential barrier of $\cong 2 \mathrm{eV}$ for isomerization from the $\tilde{X}^{2} \Pi_{u}$ level even to the lowest state $\tilde{X}^{2} B_{1}$ of the vinylidene cation making $\mathrm{H}$ migration within 52 fs extremely unlikely, and similar conclusions were drawn for isomerization of the dication in the ground state [23]. In contrast to the $\tilde{X}^{2} \Pi_{u}$ level, there are essentially no potential barriers for isomerization from the $A^{2} \Sigma_{g}{ }^{+}$state of the acetylene cation to the $\tilde{X}^{2} B_{1}$ or $\tilde{A}^{2} B_{2}$ vinylidene cation states, as shown in Fig. 1. (ii) The higher excited levels not only have small probabilities to be created but, moreover, their relaxation times are expected to be even shorter (e.g., 14 fs for the $B^{2} \Sigma_{u}{ }^{+}$state [7]) indicating that other decay mechanisms beyond isomerization, such as direct dissociation or vibronic coupling, are involved. The two isomerization processes peaked at 4.8 and $5.8 \mathrm{eV}$ correspond to the isomerization occurring in $\mathrm{C}_{2} \mathrm{H}_{2}{ }^{2+}$ and $\mathrm{C}_{2} \mathrm{H}_{2}{ }^{+}$, respectively. They proceed via different intermediate as well as final states leading to different dissociation limits, qualitatively explaining the variations in the KER.

In summary, we have traced ultrafast photoisomerization of acetylene cations in real time using intense, ultrashort pulses from FLASH. We obtained a mean isomerization time of $52 \pm 15 \mathrm{fs}$, confirming that isomerization is responsible for the ultrafast, nonradiative decay dynamics of the $A^{2} \Sigma_{g}{ }^{+}$state in the acetylene cation. Our result is in good agreement with previous predictions of $51 \mathrm{fs}$ deduced from the autocorrelation function in a time-independent experiment. The XUV-pump-XUV-probe technology opens the door for investigating the time evolution of nuclear wave packets, isomerization, elimination, and charge transfer processes in molecules under unprecedentedly clean conditions. In the future, envisaging triple coincidences between fragment ions for the acetylene dication decay, we should be able to visualize in time the structural changes, possible intermediate states, and transient structures directly by Coulomb explosion imaging.

The authors are greatly indebted to the scientific and technical team at FLASH. Support from the Max-Planck Advanced Study Group at CFEL is gratefully acknowledged. Y.H. J. acknowledges support from DFG Project No. JI 110/2, O. H., M.L., and M.F. K. from the DFG via the Emmy-Noether program and the Cluster of Excellence: Munich Center for Advanced Photonics.

[1] R. W. Schoenlein et al., Science 254, 412 (1991).

[2] A. H. Zewail, J. Phys. Chem. A 104, 5660 (2000).

[3] F. Krausz and M. Ivanov, Rev. Mod. Phys. 81, 163 (2009).

[4] G. Sansone et al., Nature (London) 465, 763 (2010).

[5] P. Rosmus, P. Botschwina, and J. P. Maier, Chem. Phys. Lett. 84, 71 (1981).

[6] G. Chambaud, R. Van den Boom, and P. Rosmus, Chem. Phys. Lett. 247, 79 (1995).

[7] J.E. Reutt et al., J. Chem. Phys. 84, 3022 (1986).

[8] G. Cooper et al., Chem. Phys. 125, 307 (1988).

[9] R. Locht and M. Davister, Chem. Phys. 195, 443 (1995).

[10] Ch. Servais and R. Locht, Chem. Phys. Lett. 236, 96 (1995).

[11] Ch. Cha, R. Weinkauf, and U. Boesl, J. Chem. Phys. 103, 5224 (1995).

[12] R. C. Gillen, B. Ostojic, and W. Domcke, Chem. Phys. 272, 1 (2001).

[13] S. Boyé-Péronne, D. Gauyacq, and J. Liévin, J. Chem. Phys. 124, 214305 (2006).

[14] T. Osipov et al., Phys. Rev. Lett. 90, 233002 (2003).

[15] A. Hishikawa et al., Phys. Rev. Lett. 99, 258302 (2007).

[16] G. Herzberg, Q. Rev. Chem. Soc. 25, 201 (1971).

[17] W. Ackermann et al., Nat. Photon. 1, 336 (2007).

[18] M. Wells and R. R. Lucchese, J. Chem. Phys. 110, 6365 (1999).

[19] J. Ullrich et al., Rep. Prog. Phys. 66, 1463 (2003).

[20] Y.H. Jiang et al., Phys. Rev. A 81, 051402 (2010).

[21] Y.H. Jiang et al., Phys. Rev. A 82, 041403 (2010).

[22] T. Pfeifer et al., Opt. Lett. 35, 3441 (2010).

[23] T. S. Zyubina et al., J. Chem. Phys. 123, 134320 (2005).

[24] R. Thissen et al., J. Chem. Phys. 99, 6590 (1993).

[25] Y.H. Jiang et al., Phys. Rev. Lett. 102, 123002 (2009).

[26] A. S. Alnaser et al., J. Phys. B 39, S485 (2006). 